\title{
Mujer y mercado laboral
}

\author{
Ana Hercilia Hamon Naranjo ${ }^{1}$
}

\section{Resumen}

En el artículo se presenta la situación de trabajo de la mujer en Colombia, en el departamento de Boyacá y en la Universidad Pedagógica y Tecnológica de Colombia durante las décadas de los sesenta y setenta. Se plantean los temas más relevantes como la dinámica de los sectores económicos, emigración, fecundidad, salarios, jornada laboral y educación. El tema de la mujer comienza a ser importante por las tendencias internacionales y nacionales, en razón de la obligatoriedad de ser incluido en todos los programas y proyectos de desarrollo.

Palabras clave: educación, jornada, mujer, trabajo, salario, sector.

\begin{abstract}
The article presents the situation of women's work in Colombia, Boyaca, and the Pedagogical and Technological University of Colombia during the sixties and seventies. It poses the most relevant matters as the dynamics in economic sectors, migration, fertility, wages, working hours and education. The subject about the woman becomes important at international and national level because of the obligation to be included in all programs and development projects.
\end{abstract}

Key words: Education, working hours, woman, work, salary, sector. 


\section{lntroducción}

Este artículo hace parte de una investigación de doctorado sobre el tema de la mujer en los años de 1960 y 1970, desarrollada en la Universidad Pedagógica y Tecnológica de Colombia. En primer lugar, se estudia la dinámica de los sectores de la economía, con el objetivo de investigar acerca de los siguientes aspectos: a) cómo fue el ingreso de la mujer en cada uno de tales sectores y b) conocer algunos factores que facilitaron el trabajo de la mujer, como la emigración, las bajas tasas de fecundidad y, quizás uno de los más importantes, su ingreso a la educación. Se justifica hacer esta investigación por la importancia del tema de género en la actualidad y se concluye que la mujer siempre ha trabajado en el hogar sin remuneración y ahora, debido a los cambios socioeconómicos, trabaja fuera del hogar aunque con inequidad.

\section{Planteamiento del problema}

Históricamente a la mujer se le asignan trabajos relacionados con servicios, y en algunos casos gratuitos, subvalorados y sin ninguna visibilidad social, aspectos que generan una discriminación a pesar de tener capacidades para desempeñarse en igualdad de condiciones que los hombres. Se justifica esta investigación porque permite conocer los factores que limitan el trabajo de la mujer y adelantar acciones que contribuyan a eliminar estos obstáculos para que, de esa manera, ella pueda laborar en términos de dignidad y equidad.

El tema de la mujer cobra relevancia en todos los niveles, llámense nacionales o internacionales, como consecuencia de las desigualdades laborales que se presentan, razón por la cual diversas instituciones y organizaciones sociales están realizando diferentes acciones para eliminar las inequidades que se presentan, con el fin de que las nuevas generaciones vivan en igualdad de condiciones. El tema mujer y trabajo es pertinente en la medida en que ella incursiona en nuevos campos laborales y además incrementa su participación, aspectos que permiten reflexionar para establecer sus condiciones reales.

\section{Desarrollo del tema}

\section{Participación de la mujer en el mercado laboral}

La condición de la mujer, destinada a ser madre y esposa, "provoca de entrada una discriminación en la educación, que posteriormente se revierte en una menor capacidad para desempeñar algunos puestos de trabajo y, por lo tanto, 
en una discriminación, a pesar de estar igualmente capacitada que el hombre. Además, la consideración básica de madre y esposa le crea dificultades de empleo, por el criterio de que si una mujer soltera ocupa un puesto de trabajo, este quedará vacante cuando aparezca un marido, o que si bien la mujer casada espera un hijo, se le van a tener que conceder demasiados permisos." (Biblioteca Salvat, 1973, p. 42).

El trabajo de la mujer se relaciona casi siempre con el de "madre, esposa, ama de casa, camarera, criada, cocinera, secretaria, recepcionista, maestra, peluquera, enfermera, auxiliar o asistente del hombre, etc." (Biblioteca Salvat 1973, p. 42). En el trabajo, solo excepcionalmente puede desempeñar cargos directivos o ejercer profesiones liberales. Las mujeres constituyen la gran mayoría del empleo doméstico, y un " $60 \%$ de los trabajos familiares sin remuneración" (Ministerio de Trabajo y seguridad Social, Transición demográfica y oferta de fuerza de trabajo en Colombia, 1986, p. 28).

Los siguientes son algunos de los fenómenos que normalmente se mencionan para explicar la mayor participación de la mujer en el mercado laboral:

Dinámica de los sectores de la economía colombiana en el período de 1960 a 1980.

Pese a la pérdida relativa del sector agrícola de 55\% en 1951 a $34 \%$ en 1980 , este continúa absorbiendo una gran cantidad de población económicamente activa. El sector industrial refleja las tendencias recesivas mundiales de la última década, con una desaceleración de $27 \%$ en el crecimiento logrado en la década de los 60. El sector de las manufacturas desacelera el $9 \%$ y el sector servicios, por el contrario, incrementa su tasa de crecimiento en $14 \%$.

La participación femenina se concentra en el sector de servicios; $58 \%$ de los trabajadores son mujeres. En este sector es donde se refleja por excelencia la división sexual de las actividades y tareas: de una parte, un alto porcentaje la conforma el trabajo doméstico, actividad tradicionalmente femenina, a la que recurren las mujeres migrantes, especialmente de baja o nula escolaridad, en oficios que desde niñas realizan en forma gratuita. Esta manera de trabajo representa la supervivencia de relaciones serviles coloniales, siendo esta población una de las minorías femeninas más oprimidas. El trabajo doméstico conforma el oficio y la labor "que las mujeres hacen como deber femenino y una responsabilidad de madres y esposas. No se considera trabajo y recibe nula o baja remuneración económica y es considerado por la mayoría de mujeres como labor aburrida, desagradecida, interminable e invisible" (Laverde, 1988, p. 310).

A partir de mediados de los años 60, la "proporción de mujeres que trabajan en el hogar ha cambiado y se presenta un gran desplazamiento al sector asalariado. Ese movimiento de mujeres desde el sector doméstico al asalariado se asemeja 
al de trabajadoras fuera del sector agrícola que ocurrió en la mayoría de países en la década de los 60: a medida que aumentan las oportunidades de trabajo en el sector moderno de la economía, los trabajadores abandonan el sector tradicional por un trabajo más productivo y más rentable" (Bustillo, 1993, p. 103). Tanto el sector doméstico como el agrícola fueron transformados por los avances tecnológicos y el debilitamiento de las barreras a la movilidad intersectorial de la mano de obra.

Otro subsector de la rama de servicios que concentra proporciones significativas del trabajo femenino es el de la educación. En Boyacá se presenta un alto crecimiento, entre otras razones, por la presencia de la Universidad Pedagógica y Tecnológica de Colombia. En este sector de servicios también están comprendidas la mayoría de actividades del gobierno, que vincula al personal femenino en cuadros administrativos medios y bajos, en los que también hay ocupaciones sociales y tradicionalmente femeninas como secretarias, mecanógrafas, recepcionistas y personal de aseo y cafetería.

Emigración. Hay cambios cualitativos en la sociedad que es importante tener en cuenta, tales como "el aumento de la proporción de la población residente en cabeceras, que pasa de un 40\% en 1951 a un 52\% en 1964" (Bustillo, 1993, p. 79). Estos nuevos patrones de distribución espacial serían causa y efecto de los cambios en la estructura económica, donde el sector primario comienza a perder importancia relativa. La migración del campo a la ciudad aporta un excedente poblacional que es captado junto con la mano de obra urbana nativa, principalmente por el sector terciario y en menor grado por el sector secundario. Este fenómeno amplía las alternativas de ocupación y estudio para la mujer.

Fecundidad. En la década de los 60, varios países desarrollados iniciaron su transición demográfica un poco después de la industrialización. La aplicación de políticas demográficas por parte de los gobiernos, tendió a disminuir la fecundidad. En Colombia, el descenso de las tasas de fecundidad antecedió al desarrollo. Hasta mediados de los 60 "la fecundidad permaneció en niveles altos, con una tasa bruta de natalidad entre 44 y 48 por mil. El descenso de la fecundidad se presentó en los años 70 en un 38 por mil; solo fue superada por Cuba, Singapur y Hong Kong y superior a la de países como costa Rica, Chile, Corea del Sur, Malasia, y Taiwán” ( Bustillo, 1993 p. 37).

El descenso de la fecundidad en Colombia se produjo como efecto de las campañas de planificación familiar y se debió a cambios socioeconómicos que efectivamente tuvieron lugar en los sectores educativo y laboral. El país presentaba en el período de "1960-1965 alrededor de 7.0 hijos por mujer y pasa en 1980 a 4.3 hijos por mujer; la fecundidad urbana disminuyó a razón de un 4\% por año entre 1968 y 1980; pasa de 6 a 3 hijos por mujer, mientras que la fecundidad rural pasa de casi 9 hijos por mujer en 1968 a 6.1 en 1980" (Bustillo, 1993, p. 38). 
El salario: El promedio de las mujeres es, a igualdad de trabajo y de capacidad, el equivalente a $2 / 3$ del salario promedio de los hombres. Las mujeres trabajan en cargos de menos responsabilidad y por eso se les paga menos que a los hombres por el mismo trabajo.

La jornada laboral. Las mujeres parecen estar destinadas en la sociedad a ser "utilizadas en el trabajo y en el hogar. Incluso entre las mujeres que trabajan se observa una fuerte discriminación en el tipo de trabajo. Actualmente, la mujer de las clases sociales inferiores ejerce un pluriempleo, trabajando fuera del hogar, llevando todo el peso de las tareas domésticas y, además, en su gran mayoría estudian" (Bustillo, 1993 p. 42).

Educación. El aumento de la participación femenina, explicado por cambio de valores, disminución de la fecundidad, cambio de patrones de unión y necesidad de complementar los ingresos del hogar, tiene que ver directamente con la mayor educación de la mujer. El total de la matrícula de los años 60 y 70 tuvo un crecimiento el cual jugó un papel importante en la oferta del trabajo y las tasas de participación. Las ocupaciones con predominio de oferta femenina son: servicios, personal administrativo, profesionales y comerciantes.

La calificación de la fuerza de trabajo, entendida como nivel de escolarización formal, se elevó entre 1960 y 1979. El recurso humano analfabeto se ha reducido en la mitad para la población femenina económicamente activa y en un $40 \%$ para el hombre. Los trabajadores con solo algunos estudios primarios se reducen a un " $10 \%$ (incremento del $3.4 \%$ para mujeres y decremento del $11 \%$ para hombres). El cambio más fuerte se registra en población económicamente activa con secundaria, que aumenta en un $164 \%$ (112\% para mujeres y $301 \%$ para hombres). La población universitaria económicamente activa con incrementos superiores al 200\% (475\% para mujeres y $273 \%$ para hombres) apenas representa el 5\% de la fuerza de trabajo" (Bustillo, 1993, p. 90).

\section{Breve análisis de la fuerza de trabajo femenino en Boyacá}

Según el censo de 1973, se observa que "el sexo masculino que participa en la actividad laboral lo hace en una cantidad superior al femenino, pero su crecimiento en la década de los 70 es estable para el hombre y creciente para la mujer" (Rodríguez, 1988, p. 40). En esta época, a la mujer se le están brindando otras oportunidades de trabajo como en el sector artesanal y otras actividades laborales como la pequeña y mediana industria.

Algo digno de resaltar es que la mujer sale a buscar trabajo en un 14\% y el hombre solo lo hace en un $9 \%$. A pesar de la vinculación de la mujer en el sector productivo, la participación del hombre sigue siendo mayor en las cabeceras municipales y en grandes industrias como cementos, cervecería, gaseosas, Sofasa, metalúrgica 
y energía, ubicadas en el corredor industrial de Boyacá, es decir, en Duitama, Sogamoso y Paipa, entre otros municipios.

Según Omaira Rodríguez, “en Boyacá en la década de los 70 existe un descenso de $1.27 \%$ continuo para la población masculina que está trabajando, en razón a que tiende a emigrar a sectores más productivos o a que son la base para prestar el servicio militar. A diferencia de los hombres, la población femenina presenta un crecimiento del $11.1 \%$, es decir, cada año hay mayor participación de la mujer en razón a mejores condiciones económicas, sociales e intelectuales" (Rodríguez, 1988, p. 40). La participación de la mujer en el mercado laboral se torna más representativa en la medida en que ella surge y tiene mayor posibilidad de realizar trabajos que años anteriores eran solo para hombres.

Al estudiar el tema del trabajo de la mujer en Boyacá no podemos desconocer aspectos socioeconómicos trascendentes como la familia, la religión y el estrato socioeconómico que, unidos a los sentimientos y tradiciones, tienen una importancia extraordinaria en el desempeño en el trabajo. En el aspecto socioeconómico destacamos las normas culturales determinadas por grupos sociales, la creciente mecanización y la tecnificación.

En Boyacá, el desempeño de la mujer en el trabajo está determinado por el medio en que se desenvuelve; aún en esta época de estudio no ha logrado su ejercicio pleno en razón a que las condiciones ofrecidas no son las más adecuadas. La mujer se desempeña como trabajadora independiente en algunos casos, aunque un alto porcentaje de mujeres son ayudantes familiares sin remuneración; se puede decir que el trabajo productivo de la mujer boyacense está económicamente subvaluado, con consecuencias negativas para ella.

\section{Situación laboral de la mujer en las entidades estatales en Tunja}

En este tema pesan varios aspectos a saber: económicos, por el sueldo devengado, y relacionados con la ocupación; culturales, basados en el pensamiento tradicional de la mujer, y originados en la familia; educativos, provenientes del seno del hogar; políticos, que se refieren a la influencia para poder ocupar cargos en entidades oficiales de la ciudad de Tunja.

El ingreso de la mujer al empleo "es considerado como uno de los medios fundamentales para eliminar la desigualdad en relación con el hombre, y el apoyo estatal a este ingreso le ha dado a ella los medios con los que logra una capacidad de negociación limitada frente a su esposo y su familia" (Acuña, 1990 , p. 130). La mujer que labora en estas empresas lo hace paralelamente con la labor de ama de casa; presta sus servicios de salud, administrativos y de servicios propiamente dichos. En el ingreso de la mujer a estas entidades se tienen en cuenta las características personales como estado civil, edad y experiencia. En relación con el tipo de actividad u ocupación dentro de la 
entidad, "el $73 \%$ se ocupan como auxiliares administrativos (secretarias, oficinistas, contabilistas, recepcionistas, etc.)" (Acuña, 1990, p. 137). Lo anterior se convierte en un ejemplo que corrobora la desigualdad laboral.

En cuanto al aspecto sociocultural, el nivel de Tunja sobresale en relación con los demás municipios por ser una ciudad estudiantil, donde por lo general los empleados tienen su bachillerato completo y algunos estudios universitarios. Este hecho no es determinante para el ingreso de la mujer a una institución gubernamental, por el predominio de las influencias políticas en Boyacá.

En el aspecto socioeconómico, el salario de la mujer es discriminatorio comparado con el del hombre; "la distribución del ingreso lo hace en mayor porcentaje en la familia, no como complemento al salario del hombre sino como factor primordial de manutención de la familia (Acuña, 1990, p. 138).

La posición de la mujer en las entidades oficiales de la ciudad de Tunja está relacionada con el cargo que desempeña. Es así como se establece una posición secundaria en relación con el hombre, aunque ellas se sienten felices con sus cargos. En otras palabras, inconscientemente se adaptan a la subordinación tradicional. Las mujeres que trabajan en estas instituciones se convierten en asalariadas y manifiestan que en la ciudad no hay más en qué ocuparse y que la necesidad las obliga. Hay más de un $60 \%$ de mujeres en las entidades oficiales, de las cuales un bajo porcentaje ocupa un cargo de jefatura.

En el año 1974 fue nombrada como gobernadora de Boyacá Susana Villarreal, quien venía de desempeñarse como embajadora en Japón. Se convirtió en la única mujer hasta ahora gobernadora; con su cariño y amabilidad transformó la gobernación; no se consideró jefe de empleado alguno, sino que se manifestó como una compañera más.

Evidentemente, la política influye en el ingreso del personal a las instituciones, ya que cada jefe político trae sus funcionarios. Como la política la hace mayoritariamente el género masculino, se ha afectado negativamente a la mujer, bien sea en su vinculación o en su jerarquización dentro del medio laboral.

Los inicios de las mujeres docentes en la Universidad Pedagógica y Tecnológica de Colombia

La primera docente que llegó a la Universidad, según los archivos históricos, fue la profesora Lucía Corsi Otálora en el año 1961 a la Facultad de Educación. Posteriormente fueron incorporándose otras mujeres en forma paulatina, principalmente a esta misma Facultad. En la década del 60 ingresaron 27 profesoras universitarias a la Facultad de Educación y tres a la de Ingeniería.

En la década del 70 se incrementa a 89 el número de mujeres docentes en la Facultad de Educación y se presenta un muy escaso aumento de cuatro docentes 
en la de Ingeniería. Algo importante es que se crean nuevos programas donde llegan bastantes mujeres docentes, como el caso de enfermería, al que ingresan 25 y la Facultad de economía y administración que nombra a trece mujeres docentes.

La mujer docente en estas décadas continúa la tendencia tradicional de serlo principalmente en educación y cuidados de la salud. Es mínimo el ingreso de personal femenino a las ingenierías, la economía y la administración. Igualmente, su categoría en el escalafón de asistente y auxiliar no le permite alcanzar niveles de Dirección de escuela o Decanatura, a excepción del programa de enfermería, cuya dirección estuvo en manos de una mujer, quien a su vez hizo presencia en el Consejo de Facultad. Una mujer continúa desempeñando el cargo de secretaria del Consejo de Facultad de Educación, un medio en el que todos sus integrantes son varones.

\section{Conclusiones}

La mujer siempre ha trabajado, inicialmente en su hogar y sin ninguna remuneración. Con el paso de los años y los cambios socioeconómicos, amplió su campo de acción fuera del hogar en diferentes actividades y, algo muy importante, mejorando cada día su nivel de educación, con el que por consiguiente obtuvo mejores oportunidades de trabajo. Fue necesario que se diera una serie de cambios económicos, sociales y culturales para que pudiera acceder a nuevas y dignas formas de trabajo. Es fundamental concientizar a nuestra sociedad de la importancia de la mujer y reconocer su papel, resaltando que puede desempeñarse en todos los campos como lo ha demostrado. El reto es seguir abriendo espacios y escalar alturas hasta las que aún no ha podido llegar.

\section{Referencias bibliográficas}

Acuña S., M. A. (1990). Situación laboral de las mujeres en las entidades estatales en Tunja. Trabajo de grado, Administración de empresas, Universidad Pedagógica y Tecnológica de Colombia, Tunja, Colombia.

Bustillo, I. (1993). Productividad y trabajo de la mujer. Revista CEPAL, 51, 100-115. Santiago de Chile, Chile.

Laverde, A. (1988). Representaciones cognitivas del trabajo en un grupo de mujeres de estrato bajo en Bogotá. Universitas Humanistica. 17 (29), 89-122. Bogotá, Colombia. 


\section{Revista de}

investigaciones UNAD

Volumen 09. Número 3. Diciembre 2010

Ministerio de trabajo y seguridad social .Dirección General del Servicio Nacional de Empleo. (1986). Transición demográfica y oferta de fuerza de trabajo en Colombia. Bogotá, Colombia.

Rodríguez, O. (1988). Fuerza de trabajo femenino en Boyacá. Trabajo de grado, Licenciatura en Ciencias Sociales, Universidad Pedagógica y Tecnológica de Colombia, Tunja, Colombia. 